\title{
Spatial distribution of particles sputtered from single crystals by gas cluster ions
}

Nazarov , A.V.

2017-01-22

Nazarov , A V , Chernysh, V S , Nordlund, K, Djurabekova, F \& Zhao , J 2017 , ' Spatial distribution of particles sputtered from single crystals by gas cluster ions ' , Nuclear Instruments \& Methods in Physics Research. Section B: Beam Interactions with Materials and Atoms , vol. 406 , pp. 518-522 . https://doi.org/10.1016/j.nimb.2017.01.014

http://hdl.handle.net/10138/308791

https://doi.org/10.1016/j.nimb.2017.01.014

cc_by_nc_nd

acceptedVersion

Downloaded from Helda, University of Helsinki institutional repository.

This is an electronic reprint of the original article.

This reprint may differ from the original in pagination and typographic detail.

Please cite the original version. 
Author"s final preprint of paper published as

A. Nazarov, V. S. Chernysh, K. Nordlund, F. Djurabekova, and J. Zhao, Spatial distribution of particles sputtered from single crystals by gas cluster ions, Nucl. Instr. Meth. Phys. Res. B 406, 518 (2017). 


\title{
SPATIAL DISTRIBUTION OF PARTICLES SPUTTERED FROM SINGLE CRYSTALS BY GAS CLUSTER IONS
}

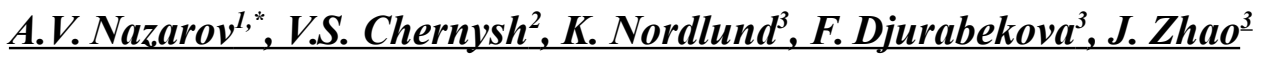 \\ ${ }^{1}$ Skobeltsyn Institute of Nuclear Physics, Lomonosov Moscow State University, Russian \\ Federation \\ ${ }^{2}$ Faculty of Physics, Lomonosov Moscow State University, Russian Federation \\ ${ }^{3}$ Department of Physics, University of Helsinki, Finland
}

\begin{abstract}
The results of molecular dynamics simulations of the bombardment of the $\mathrm{Cu}(100)$ and $\mathrm{Mo}$ (100) single-crystals by $10 \mathrm{keV}$ Ar cluster ions of different sizes are presented in this paper. Spatial distributions of sputtered material were calculated. The anisotropy of the angular distributions of sputtered atoms was revealed. It was found that the character of the anisotropy is different for $\mathrm{Cu}$ and Mo targets. The reasons leading to this anisotropy are discussed according to the dependences of the angular distributions on the cluster size and on the target material.
\end{abstract}




\section{Introduction}

The spatial distributions of the particles sputtered from solid targets by gas cluster ion beams (GCIB) are important for understanding the mechanisms of sputtering phenomenon as well as for a wide range of technology applications such as surface smoothing. The angular distributions of sputtered material are a determinative factor for surface polishing processes $[1]$.

The angular distributions of atoms sputtered by GCIB for a number of target materials are found to have maximum far from the normal to the surface (so-called lateral distributions). Such distributions were observed experimentally and in molecular dynamics (MD) simulations [2-3]. However, recently it was found experimentally, that for some materials (Mo, W) the angular distributions have significantly higher sputtering yield in the direction of the normal to the surface in comparison to $\mathrm{Cu}$, In and $\mathrm{Cd}[4]$.

Most of the published experimental data on the angular distributions of atoms sputtered by GCIB from the metals is related to the polycrystalline targets. However, the simulations are more often performed with single crystal lattices. In a recent paper [5] the anisotropy over the azimuthal angle of the spatial distributions of $\mathrm{Ag}$ and Mo atoms sputtered from single-crystal targets with $10 \mathrm{keV}$ Ar clusters has been found. This is surprising because the crystalline structure in the impact area is destroyed during the cluster impact on the target surface.

In order to understand the reasons that lead to the spatial anisotropy of the angular distributions of sputtered material the MD simulations of sputtering of $\mathrm{Cu}$ and Mo single crystals by Ar clusters of different sizes were performed in this work.

\section{Methods}

The MD simulations of the $10 \mathrm{keV}$ Ar cluster bombardment of $\mathrm{Cu}(100)$ and $\mathrm{Mo}(100)$ single crystals were performed at room temperature. The size of the clusters varied from 50 to 1500 
atoms per cluster. The incident direction of the cluster ions was normal to the target surface. The simulations were performed with the PARCAS code [6-8]. The Lennard-Jones potential was used for the Ar-Ar interactions, the EAM potentials [9-10] for the Mo-Mo and $\mathrm{Cu}-\mathrm{Cu}$ interactions and the density-functional theory based pair-specific repulsive potentials [11] for the Ar-Mo and Ar-Cu interactions. The size of the simulation cell was 50x50x40 unit cells for $\mathrm{Cu}$ target and $48 \times 48 \times 48$ unit cells for Mo target with periodic boundary conditions in $x$ and $y$ directions.

From three to seven hundred impacts of Ar clusters, depending on the cluster size, were simulated for the $\mathrm{Cu}(100)$ target. For the $\mathrm{Mo}(100)$ target, five - seven hundred impacts were simulated. However, we note that more simulations are needed for the $\operatorname{Mo}(100)$ target to acquire good statistics because of the much lower sputtering yield.

The simulated time varied from $15 \mathrm{ps}$ to $30 \mathrm{ps}$, depending on the cluster size, so that all the redepositing processes are finished. Each impact was simulated with virgin target lattice, so that the evolution of surface morphology due to the ion bombardment is not taken into account.

\section{Results and discussion}

The simulation results showed that many atoms are sputtered as dimers, trimers and larger clusters that can rotate around the center of mass, which is in a good agreement with the results by other authors [5]. The number of such sputtered clusters for the $\mathrm{Cu}$ target is much higher than that for the Mo one. For instance, during the cluster bombardment by $\operatorname{Ar}_{100}$ of the $\mathrm{Cu}$ target, $55 \%$ of the sputtered atoms left the surface as clusters, while the same number for the Mo target was only $27 \%$. Moreover, the maximum size of sputtered $\mathrm{Cu}$ clusters is twice as large as of the Mo ones.

Fig. 1 represents the polar and azimuthal distributions of the sputtered material deposited on the hypothetical hemispherical collector. The polar axis of the plot corresponds to the 
azimuthal angle of the sputtered atom velocity and the radial axis corresponds to the polar angle measured from the target surface normal. For atoms sputtered as clusters, the velocity of the center of mass was taken into account instead of the velocity of individual atoms. The distinct anisotropy of the distributions shown in Fig. 1 over the azimuthal angle, the so-called "spots", is clearly visible for both Mo and Cu targets, at least for smaller sizes of Ar clusters. Note that the similar distributions were observed for bombardment of $\operatorname{Ag}(100)$ surface by $\operatorname{Ar}$ clusters [5]. The centers of spots visible in Fig. 1 are located in the (110) plane for the Mo target with the bcc lattice and in the (100) plane for the $\mathrm{Cu}$ target with the fcc lattice. These planes are perpendicular to the target surface, and it should be noted that these planes are the close-packed ones for $\mathrm{Mo}$ and $\mathrm{Cu}$, respectively.

We point out one more feature of the spatial distributions: the spots for Mo can be clearly observed for all cluster sizes that lead to any reasonable sputtering (up to 500 atoms per cluster), while for the $\mathrm{Cu}$ target it becomes imperceptible for sizes more than 300 atoms per cluster.

For more detailed analysis, the polar angular distributions in the azimuthal direction of the spots and in the middle between the spots are plotted. Fig. 2 shows the areas that are used to build such distributions for the exemplary case of the $\mathrm{Ar}_{100}$ cluster and the Mo target. The angle width of these areas is 20 degrees. These distributions for Mo and $\mathrm{Cu}$ targets are presented in Fig. 3 and Fig. 4, respectively.

It is important to mention that one should not compare these angular distributions with the distributions obtained experimentally with the semi-cylindrical collector as done in $[2,4]$. Such distributions are presented here in order to illustrate the specific features of the shown anisotropy comparing the cases of different target materials and incident cluster sizes.

The polar distributions of the sputtered material in the azimuthal direction corresponding to the maximum of the spots as well as the distribution between the spots are further away from 
the surface normal in case of larger Ar cluster for both $\mathrm{Cu}$ and Mo targets. This means that this kind of anisotropy cannot be considered as the Wehner spots, as mentioned in [5]. This conclusion is based on the fact that numerous experiments have shown that the angular positions of Wehner spots are determined only by the crystal structure of the irradiated surface. It was also found that the angular position of Wehner spots does not depend on the type and energy of bombarding ions [12-18].

In order to understand the nature of the anisotropy of the spatial distributions of sputtered material the distributions similar to Fig. 3 and Fig. 4 were calculated for a wide range of cluster sizes (50-1500 atoms per cluster for $\mathrm{Cu}$ target and 44-500 atoms per cluster for Mo target). From these simulations, the dependences of the polar position of the maximum of these distributions on the cluster size were calculated (see Fig. 5 and Fig. 6). One can see from these figures that the polar position of the distribution maximum shifts further from the surface normal with the increase of the cluster size for both Mo and $\mathrm{Cu}$ targets.

The polar position of the maximum in the polar angular distributions in the azimuthal direction of the spots and in the middle between the spots reaches its highest value of about 67.5 degrees at the incident cluster size of 600 atoms per cluster for the $\mathrm{Cu}$ target. However, these two curves, as seen from Fig. 5 coincide already for the Ar clusters of 300 atoms. Thus, the result shows that anisotropy of the spatial distribution of sputtered particles cannot be observed for most of $10 \mathrm{keV}$ Ar cluster sizes larger than 300 atoms, which make a noticeable contribution into the sputtering of $\mathrm{Cu}$. At the same time, the polar position of the maximum in the polar angular distributions in the azimuthal direction of the spots and in the middle between the spots found for Mo, as seen from Fig. 6 significantly differs from similar dependences for $\mathrm{Cu}$. First of all, the curves plotted in Fig. 6, do not intersect. Secondly, the values of the polar position of the maximum in the spot are higher than the corresponding values in between spots. These results indicate that in the case of Mo (100) single-crystal 
sputtering, the anisotropy can be observed for all sizes of incident clusters that make any contribution into sputtering.

Without discussing in detail the mechanisms responsible for the specific angular distributions of material sputtered under cluster ion bombardment, it can be noted that for both $\mathrm{Cu}$ and Mo targets, the maximum of the polar angular distributions shifts from direction of the normal to the surface toward off-normal direction with increase in the cluster size. Detailed analysis of the processes responsible for the observed specific angular distributions of sputtered particles under cluster ion bombardment was carried out in [5]. The interaction of the atoms constituting the cluster with the walls of the crater plays an important role in the formation of the flux of the sputtered atoms. The authors note that a large lateral component of the force, appearing in the compression/decompression during initial phase of cluster-target interaction, affects the surrounding medium. This process leads to a faster increase in the crater diameter as compared to a change of its depth [5]. As it was pointed in [5], this process leads to strong side jet of Ar atoms. Apparently, a flux of such atoms should be isotropic in the azimuthal directions, however the density of target atoms that form the crater walls in different azimuthal directions is different. This density has the maximum in the close-packed directions and the minimum in between. As mentioned above, in case of the Mo target such closepacked planes are (110) and (100) for $\mathrm{Cu}$. It is obvious that the elasticity of the crater walls varies in the azimuthal directions accordingly. This factor has in our opinion a decisive influence on the formation of the flux of the sputtered particles. This assumption is confirmed by the results of experimental and computer simulations studies of the shapes of craters formed in Si single crystals under bombardment by Ar clusters [19]. The AFM measurements showed that the $\mathrm{Si}(100)$ craters exhibit four-fold symmetry, while the shape of craters on a Si(111) surface shows three-fold symmetry. 
In addition, we note one more issue. Since the elastic modulus of $\mathrm{Cu}$ is much smaller than that for Mo, the anisotropy of the spatial distribution of sputtered particles becomes negligible in case of $\mathrm{Cu}$ for the clusters of relatively small sizes.

The collapse of the cluster ion (atomization) during the impact plays an important role in the process of cluster-target interaction, since such an impact destroys completely the crystal structure of the surface at the impact spot.

Thus, the analysis shows that sputtering by atomic ions and cluster ions are significantly different. It is well known that in the case of sputtering by atomic ions, the anisotropy of the spatial distribution of the sputtered material is determined by the crystal structure of the irradiated material in the region of the cascade of atomic collisions. In case of cluster bombardment, the crystal structure outside the impact region determines the spatial distribution of sputtered particles.

\section{Conclusions}

Cluster ion bombardment of $\mathrm{Cu}$ and Mo surfaces led to the sputtering of these materials to a significant extent in shape of dimers, trimers and larger clusters. The number of such sputtered clusters for the $\mathrm{Cu}$ target was found to be much higher than for the Mo target. Moreover, the maximum size of such sputtered cluster is also larger for the $\mathrm{Cu}$ target than for the Mo one.

The distinct anisotropy of the sputtered atoms distributions over the azimuthal angle is observed for a wide range of cluster sizes for the $\mathrm{Mo}$ and $\mathrm{Cu}$ single-crystal targets. This anisotropy has a "spot" character. The spots are located in low-index crystallographic planes. The position of the spots over the polar angle depends on the impacting cluster size. This anisotropy cannot be considered as the Wehner spots because the spots polar positions in case 
of sputtering by the gas cluster ion beam depend on the cluster size, whereas the Wehner spots positions always match close-packed crystallographic axis.

\section{Acknowledgments}

This work was supported by the Ministry of Science and Education of the Russian Federation, agreement no. 14.607.21.0072, project no. RFMEFI60714X0072. 


\section{References}

1. I.Yamada, Appl. Surf. Sci., 310 (2014), 77-88.

2. N. Toyoda, H. Kitani, N. Hagiwara, T. Aoki, J. Matsuo, I. Yamada,Mater. Chem. Phys., 54(1998), 262-265.

3. Z. Insepov, I. Yamada, Nucl. Instrum. Meth. B, 99 (1995), 248-252.

4. V.S. Chernysh, A.E. Ieshkin, Yu.A. Ermakov,Appl. Surf. Sci., 326 (2015), 285-288.

5. D. Maciazek, M. Kanski, L. Gaza, B. J. Garrison, Z. Postawa, J. Vac. Sci. Technol., B 34(2016), 03H114.

6. K. Nordlund, M. Ghaly, R.S. Averback, M. Caturla, T. Diaz de la Rubia, J. Tarus, Phys. Rev. B. 57 (13) (1998), 7556 - 7570.

7. M. Ghaly, K. Nordlund, R. S. Averback, Phil. Mag. A, 79(4) (1999), 795.

8. K. Nordlund, Comput. Mater. Sci., 3 (1995), 448.

9. Michael J. Sabochick, Nghi Q. Lam, Phys. Rev. B, 43(7) (1991), 5243.

10. E. Salonen, T. Järvi, K. Nordlund, J. Keinonen, J. Phys. Cond. Matt., 15 (2003), 58455855

11. K. Nordlund, N. Runeberg, D. Sundholm, Nucl. Instr. Meth. Phys. Res. B, 132 (1997), 4554.

12. G.K. Wehner, J. Appl. Phys., 26 (1955), 1056.

13. G.K. Wehner, Phys. Rev., 102 (1956), 690.

14. V.E. Yurasova, N.V. Pleshivtsev, I.V. Orfanov, JETP, 37 (1959), 966.

15. M.W. Thompson, Philos. Mag., 4 (1959), 139

16. B. Perovic, Inst. Nucl. Sci. "Boris Kidrich", 11 (1961), 37. 
17. R.H. Silsbee, J. Appl. Phys., 28 (1957), 1246

17. R.S. Nelson, M.W. Thompson, H. Montgomery, Philos. Mag., 7 (1962), 1385

18. L. P. Allen, Z. Insepov, D. B. Fenner, C. Santeufemio, and W. Brooks, K.

19. S. Jones, I. Yamada.J. Appl. Phys., 71 (2002) 
Figure 1. The polar and azimuthal distributions of sputtered material: a) Mo target, $\operatorname{Ar}_{44}$ clusters; b) Mo target, $\mathrm{Ar}_{300}$ clusters; c) Mo target, $\mathrm{Ar}_{500}$ clusters; d) Cu target, $\mathrm{Ar}_{50}$ clusters; e) $\mathrm{Cu}$ target, $\mathrm{Ar}_{100}$ clusters; f) $\mathrm{Cu}$ target, $\mathrm{Ar}_{900}$ clusters

Figure 2. The areas used to build polar distributions in the azimuthal direction of the spots and in the middle between the spots.

Figure 3. The polar angular distributions of material sputtered with the $\operatorname{Ar}_{44}$ clusters $(a, b)$ and $\operatorname{Ar}_{500}$ clusters (c, d) from the Mo (100) target in the azimuthal direction of the spots (a, c) and in the middle between spots $(b, d)$.

Figure 4. The polar angular distributions of material sputtered with the $\operatorname{Ar}_{50}$ clusters $(a, b)$ and $\operatorname{Ar}_{900}$ clusters $(\mathrm{c}, \mathrm{d})$ from the $\mathrm{Cu}(100)$ target in the azimuthal direction of the spots $(\mathrm{a}, \mathrm{c})$ and in the middle between spots $(b, d)$.

Figure 5. Dependence of the position of the maximum of the polar angular distributions of material sputtered from the $\mathrm{Cu}(100)$ target from the Ar cluster size.

Figure 6. Dependence of the position of the maximum of the polar angular distributions of material sputtered from the Mo (100) target from the Ar cluster size. 

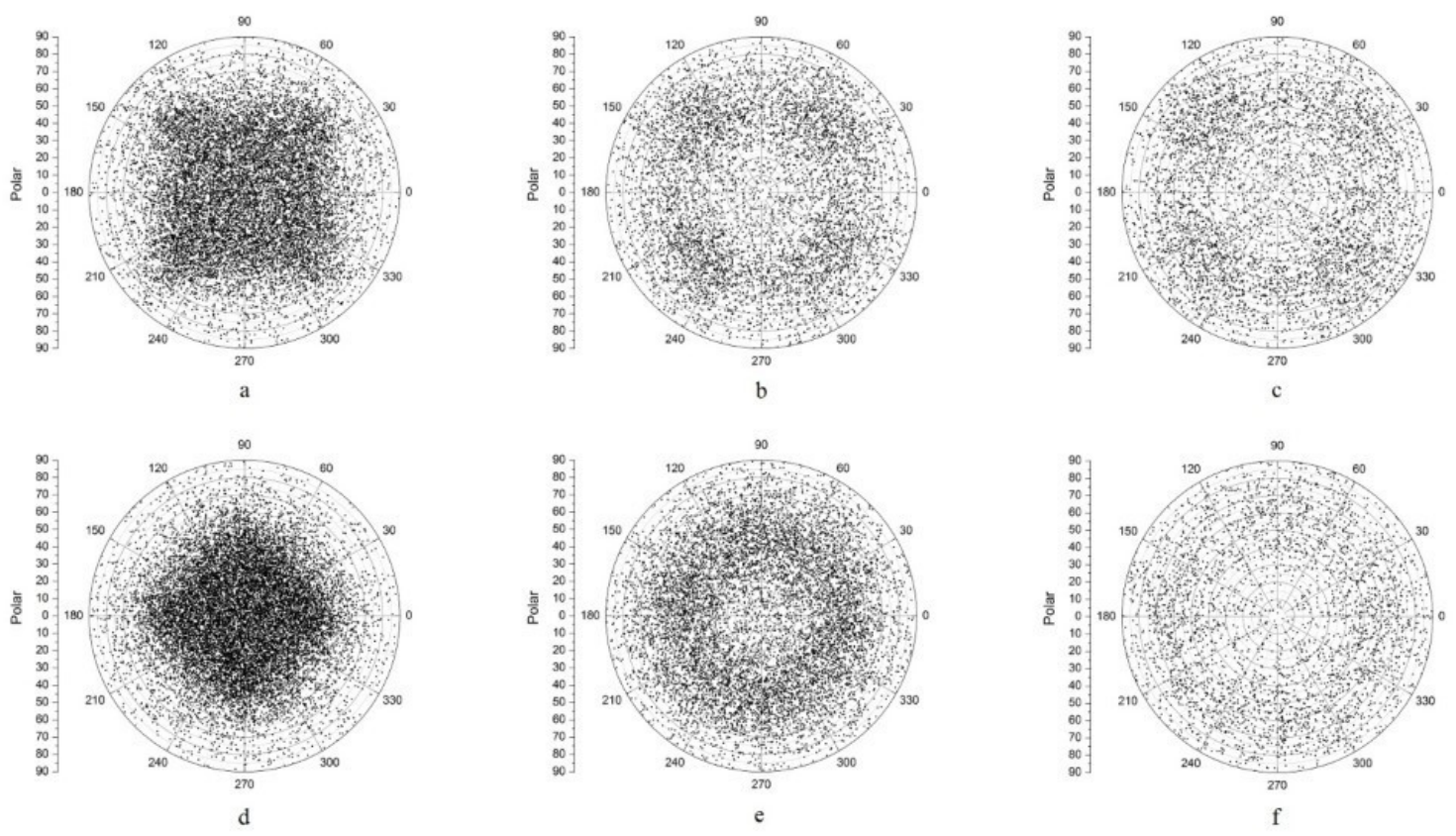


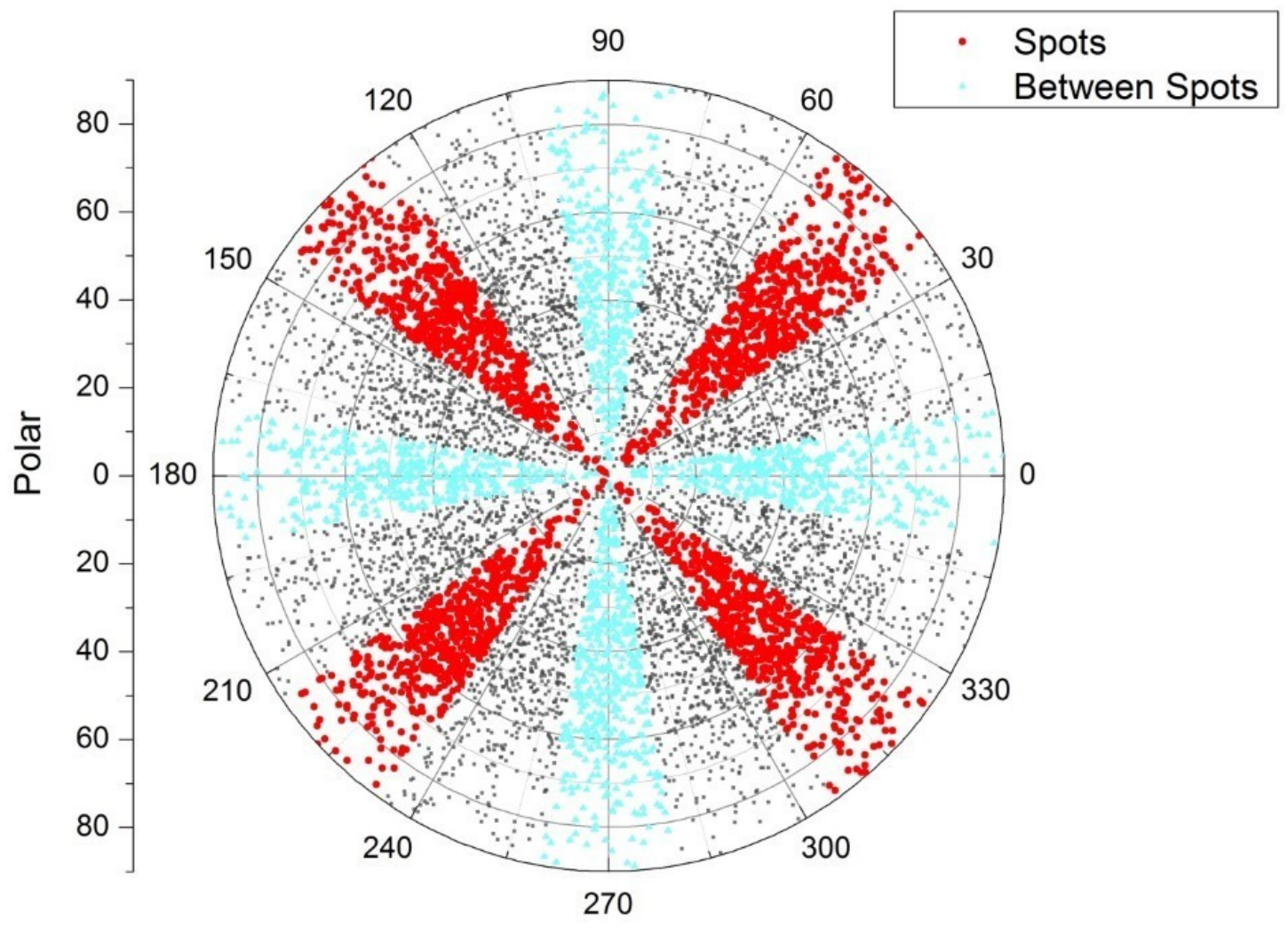

2 


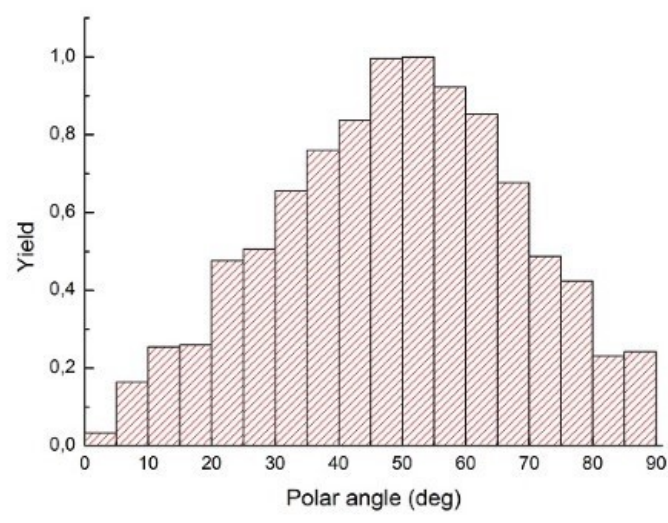

a

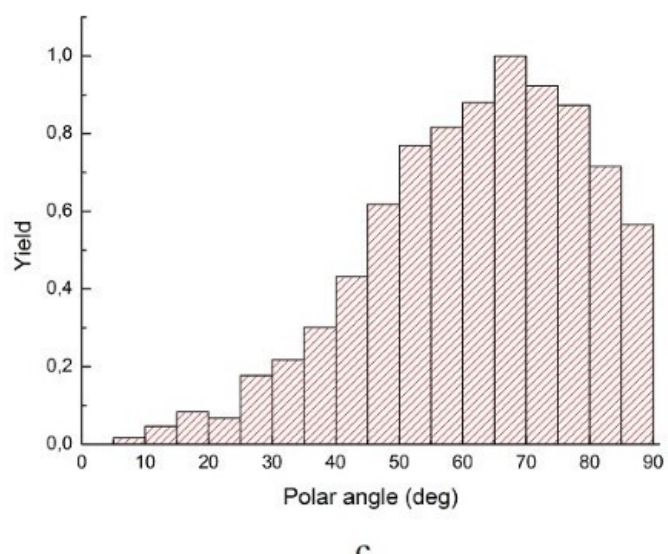

c

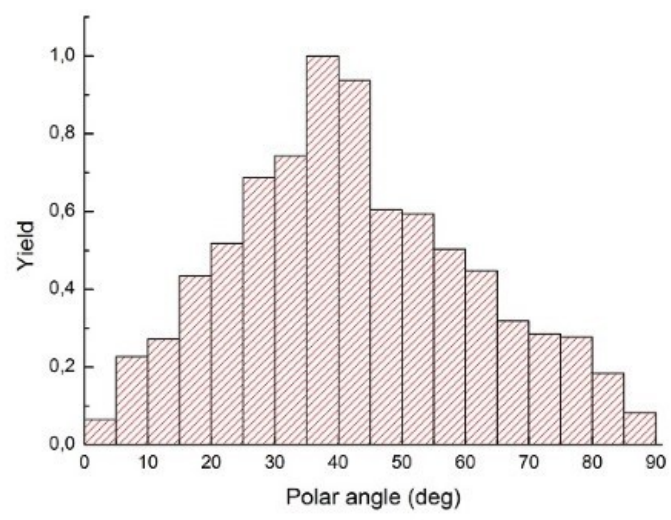

b

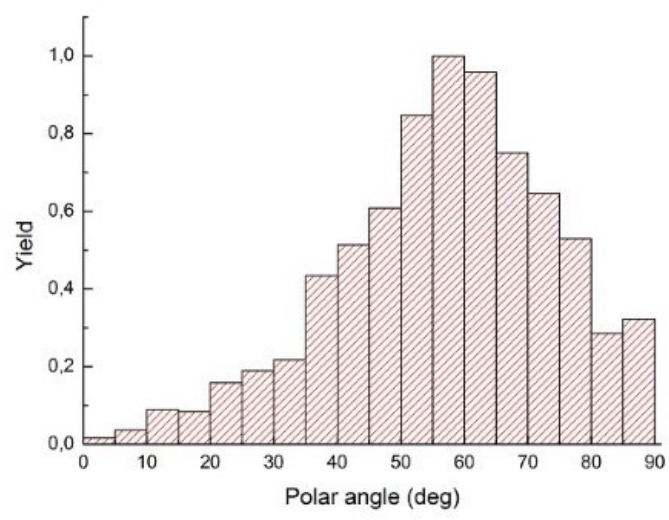

d

3 


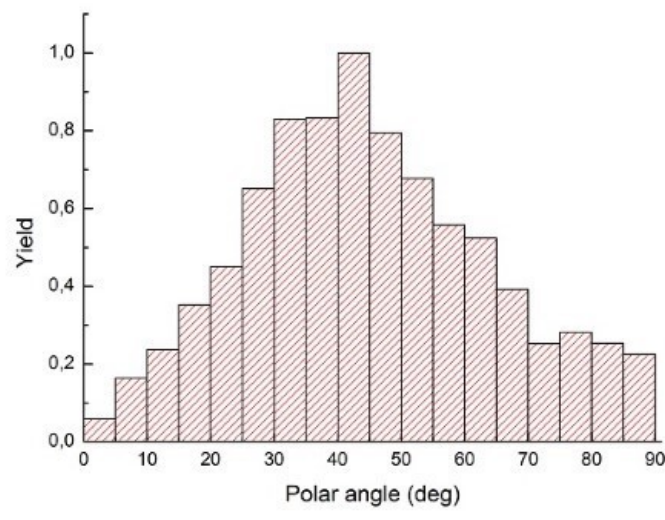

a

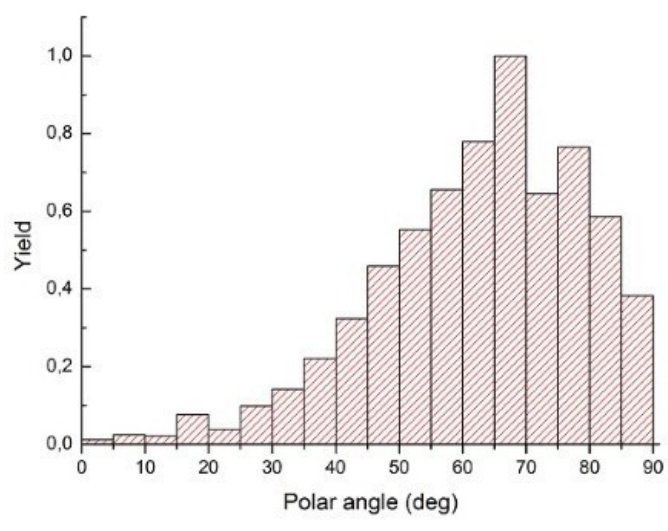

c

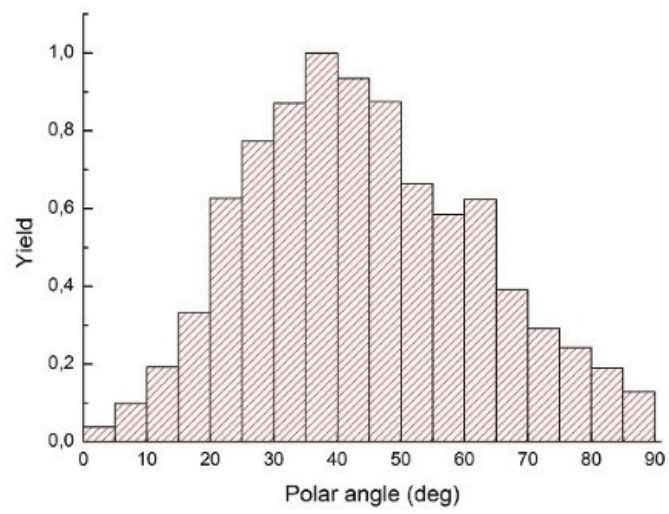

b

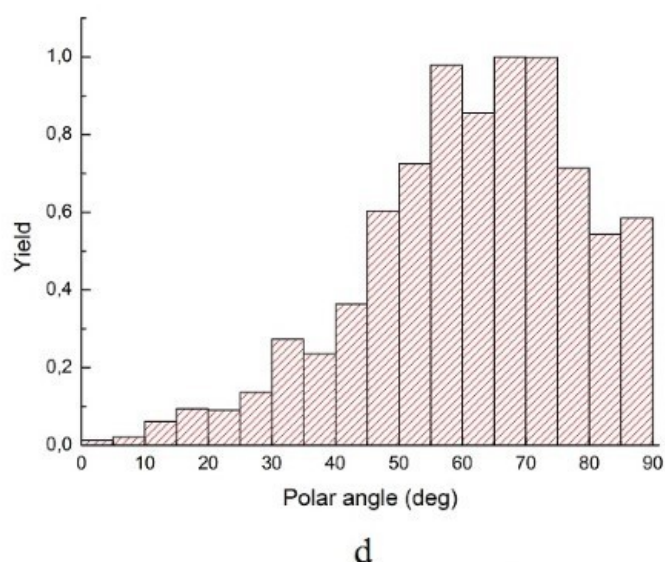

d

4 


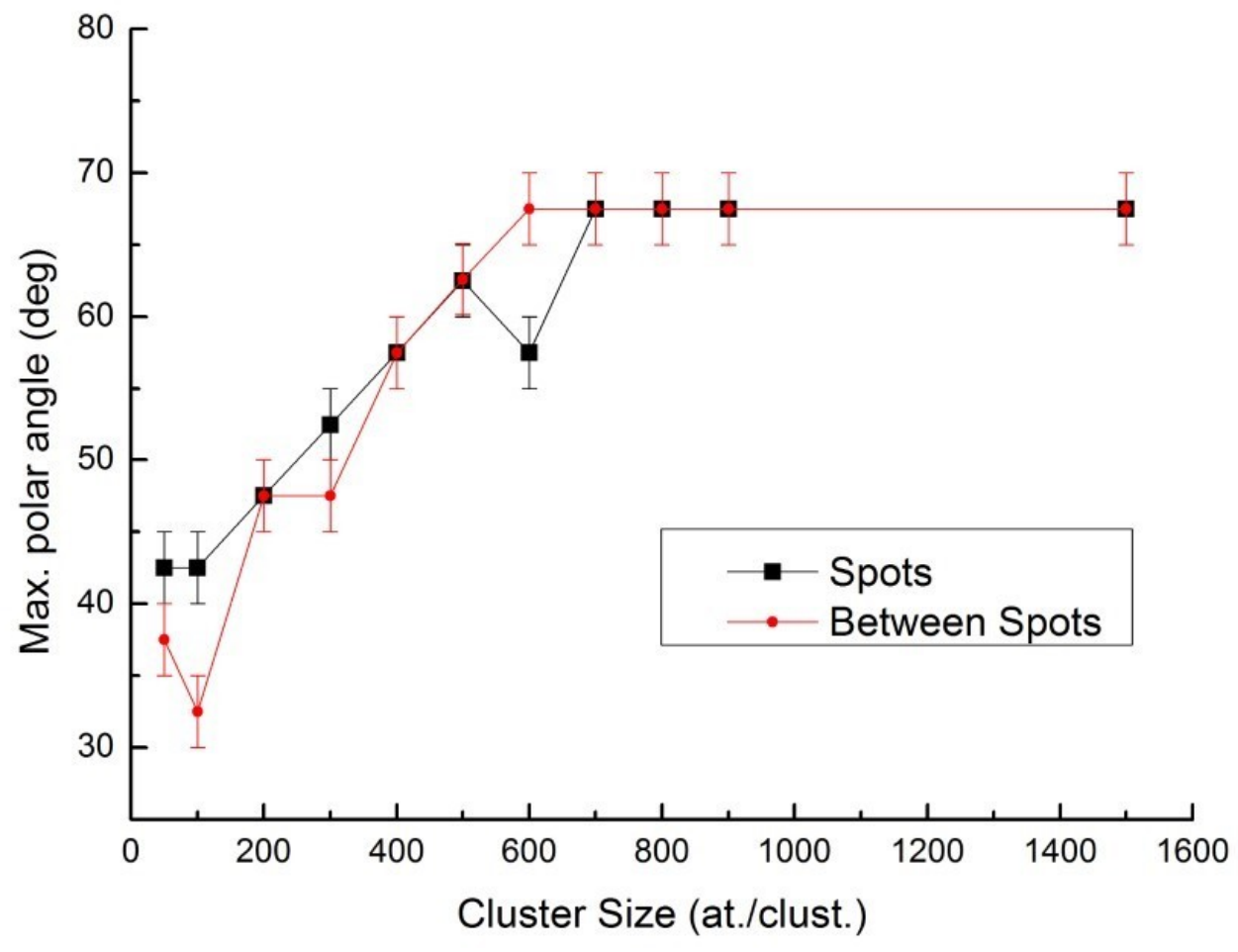

5 


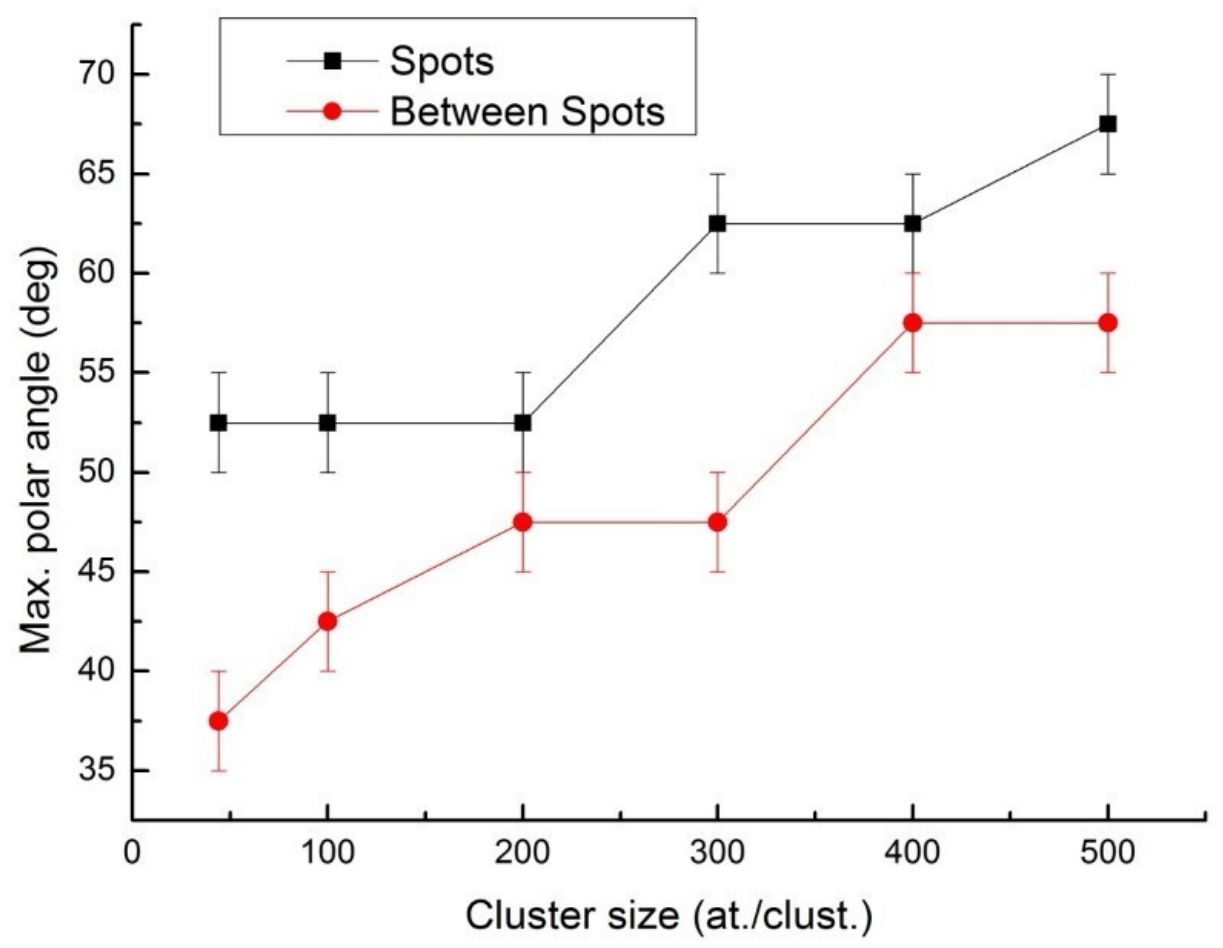

6 